\title{
TITLE:
}

\section{Cortisol analysis of hair of captive chimpanzees (Pan troglodytes).}

\section{AUTHOR(S):}

Yamanashi, Yumi; Morimura, Naruki; Mori, Yusuke; Hayashi, Misato; Suzuki, Juri

\section{CITATION:}

Yamanashi, Yumi ... [et al]. Cortisol analysis of hair of captive chimpanzees (Pan troglodytes).. General and comparative endocrinology 2013, 194: 55-63

ISSUE DATE:

2013-09-05

URL:

http://hdl.handle.net/2433/179310

\section{RIGHT:}

@ 2013 Elsevier Inc.; この論文は出版社版でありません。引用の際には 出版社版をご確認ご利用ください。; This is not the published version. Please cite only the published version. 
Running head: Hair cortisol analysis of chimpanzees

22

Title: Cortisol analysis of hair of captive chimpanzees (Pan troglodytes)

\author{
YumiYamanashi $^{\mathrm{ab}}$

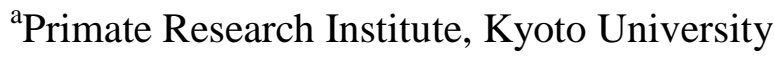 \\ ${ }^{\mathrm{b}}$ Japan Society for Promotion of Science \\ NarukiMorimura $^{\mathrm{c}}$ \\ ${ }^{\mathrm{c}}$ Wildlife Research Center, Kyoto University \\ Yusuke Mori ${ }^{\mathrm{c}}$ \\ ${ }^{c}$ Wildlife Research Center, Kyoto University \\ Misato Hayashi ${ }^{\mathrm{a}}$

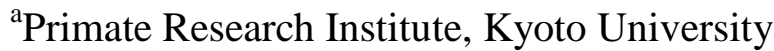 \\ JuriSuzuki $^{\text {a }}$ \\ ${ }^{\mathrm{a}}$ Primate Research Institute, Kyoto University
}

\title{
Address
}

${ }^{a}$ Primate Research Institute, Kyoto University, 41-2, Kanrin, Inuyama City, Aichi, 484-8506, Japan

${ }^{\mathrm{b} J a p a n}$ Society for Promotion of Science, Kojimachi Business Center Building, 5-3-1

Kojimachi, Chiyoda-ku, Tokyo, 102-0083, Japan

${ }^{\mathrm{c} W i l d l i f e}$ Research Center, Kyoto University, 3rd Floor, 2-24 Tanaka-Sekiden-cho, Sakyo, Kyoto, 606-8203, Japan 
Running head: Hair cortisol analysis of chimpanzees

26 yamanashi@wrc.kyoto-u.ac.jp

3rd Floor, 2-24 Tanaka-Sekiden-cho,

Sakyo, Kyoto, 606-8203, Japan 
Running head: Hair cortisol analysis of chimpanzees

\section{Abstract}

In addition to behavioral evaluations, stress assessments are also important for measuring animal welfare. Assessments of long-term stress are particularly important given that prolonged stress can affect physical health and reproduction. The use of hair cortisol as a marker of long-term stress has been increasing, but there has not yet been any report on the use of such methods with chimpanzees. Therefore, the purpose of this study was to establish and validate a methodology for analyzing hair cortisol in captive chimpanzees. In the first experiment, hair was removed from the arms of nine chimpanzees living in the Kumamoto Sanctuary (KS) and the regrown hair was sampled 3 months later. Fecal samples were collected periodically during the hair-growth period. The results showed that hair cortisol level was positively correlated with the rate of receiving aggression. Although the correlation between hair and fecal cortisol levels was not significant, the individual with the highest hair cortisol concentration also had the highest fecal cortisol concentration. These results suggest that hair cortisol may reflect long-term stress in chimpanzees. In the second experiment, we investigated the physiological factors affecting hair cortisol concentrations. We cut hair from the arms, sides, and backs of 25 chimpanzees living at the KS and the Primate Research Institute. The results revealed that cortisol varied based on source body part and hair whiteness. Therefore, we recommend that hair should always be collected from the same body part and that white hair should be avoided as much as possible.

\section{Keywords}

Hair cortisol; Chimpanzees; Welfare; All-male group; Aggression 
Running head: Hair cortisol analysis of chimpanzees

\section{Introduction}

Many chimpanzees are kept in captive environments around the world. A total of 325 chimpanzees were living in 51 Japanese facilities including zoos, sanctuaries, and laboratories as of 2013 [23]. In the US, 1,970 chimpanzees were living in zoos, sanctuaries, laboratories, and private houses as of 2012 [44]. A total of 1,032 chimpanzees were registered in the studbook of the European Associations of Zoos and Aquariums [12]. Chimpanzees live in a physically and socially complex environment in their wild habitats, but captive environmentsdiffer greatly from their original habitats [45]. Despite the large number of captive chimpanzees and increasing attention toward animal welfare, little is known about the link between stress and the environment of chimpanzees living in such captive environments. Understanding animal stress is important, and long-term assessments of stress are particularly important because prolonged stress often has deleterious effects on behaviors, reproduction, and physical and mental health $[11,13,53]$. However, practical constraints often prevent assessment of long-term stress in many individuals. Therefore, it is important to develop practical ways of estimating long-term stress in chimpanzees.

Cortisol is a steroid hormone released by the adrenal cortex, and its production usually increases in reaction to stressors [19]. Cortisol helps mobilize energy during stress by influencing the metabolism of sugar in the direction of increasing blood glucose levels [53]. Therefore, it is often used as a marker of stress. Cortisol assessment in chimpanzees has been performed using blood, urine, feces, and saliva [3-5, 17, 24, 30, $40,57]$. However, the use of these types of samples for estimating long-term stress presents several difficulties. For example, cortisol responds to circadian rhythms, and the peak cortisol level is found just before arousal from sleep [47]. Additionally, the 
Running head: Hair cortisol analysis of chimpanzees

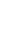

time course of cortisol excretion in chimpanzees is short: peak cortisol levels were detected in fecal samples $22 \mathrm{hrs}$ after injection of ${ }^{3} \mathrm{H}$-labeled cortisol [5]. Thus, samples should be collected at multiple times. Additionally, repeated blood-sample collection can itself be stressful for animals. Recently, an increasing number of studies have validated the use of hair for stress measurement $[2,9,15,18,28,32,49,58]$. For example, Davenport et al. (2006) found a significant correlation between hair and saliva cortisol concentrations and showed that the hair cortisol concentrations in a group of rhesus macaques increased after their relocation to another institute. Hair cortisol levels have an advantage for the assessment of long-term stress insofar as it has been hypothesized that they reflect an accumulation over a period of time. Additionally, chimpanzees often form good relationships with humans [34], which may enable us to collect hair samples relatively easily. However, there have been no reports to date on hair cortisol measurements in chimpanzees. Considering that the manner in which substances such as cortisol are incorporated into hair is not fully understood [22, 25] and that there are variations in physiology and hair features between species, it is necessary to validate the use of hair cortisol as a stress marker for each species separately and to modify the methodology appropriately.

Hence, this study aimed to investigate the validity of hair cortisol as a marker of long-term stress in chimpanzees and to examine how physiological factors such as the body part from which the hair was taken influence hair cortisol concentrations. living at the Primate Research Institute, Kyoto University (PRI) $(N=13)$ and the 
Running head: Hair cortisol analysis of chimpanzees

Kumamoto Sanctuary, Kyoto University $(\mathrm{KS})(N=13)$. Basic information about each subject is presented in Table 1 .

At the PRI, the subject chimpanzees lived in two mixed-sex groups in an outdoor enclosure that connected to several inside rooms. The outdoor enclosure was separated into two compartments: one was a $700-\mathrm{m}^{2}$ outdoor compound with $15-\mathrm{m}$-high climbing frames, a small stream and numerous trees; the other was a $250-\mathrm{m}^{2}$ outdoor compound with climbing frames and two small streams [35, 41]. Chimpanzees could freely access the outdoor enclosure and inside room at all times. The chimpanzees were fed seasonal fruits and vegetables, along with monkey pellets three times per day. During the experimental period, feeding-enrichment items were provided between meals on a few occasions, and cognitive experiments were conducted on some individuals $[34,36,43]$.

The KS was the first chimpanzee sanctuary in Japan and is now operated by the Wildlife Research Center, Kyoto University [38]. At the KS, the subject chimpanzees lived in all-male groups. All-male groupingare not observed in wild chimpanzees, but are often formed in captivity to solve the problem of surplus animals [10, 20]. There were three all-male groups in the sanctuary, and each subject belonged to one of these groups. These individuals lived in an outdoor cage that connected to inside rooms. All outdoor cages were covered with iron mesh fences,and were about $120 \mathrm{~m}^{2}$ in size and $3.8 \mathrm{~m}$ in height. Some grass and shrub vegetation was present on the ground. Climbing structures and several feeding devices were present in the cages for environmental enrichment. Indoor rooms were small cells $4 \mathrm{~m}^{2}$ in size and $2.7 \mathrm{~m}$ in height. During the experimental period, the subjects were isolated in indoor rooms from evening until the next morning, but were able to communicate with neighboring individuals by visual and 
Running head: Hair cortisol analysis of chimpanzees

physical contact through the iron bars $[38,39]$. KS chimpanzees were fed seasonal fruits and vegetables three times a day. Additionally, various enrichment efforts were made every day [29]. During the period of this study, as part of a social enrichment program, group members were changed on a daily basis. For example, one day, 15 chimpanzees were divided into two groups of 10 and five members. On another day, 15 chimpanzees were divided into three groups of five members each. Additionally, Toon and Kenny, two of the subjects in this study, arrived from a zoo in June 2008 and were initially integrated into an all-male group on June $25^{\text {th }}$, 2008. Thus, the social stimulation available to chimpanzees at KS was greater than that at the PRI, which did not offer such social variation during the period of this study.

\section{Experiment 1}

In Experiment 1, we examined whether the cortisol concentration in hair was correlated with that in feces and with aggressivebehaviors.

\subsubsection{Study site and subjects}

\subsection{Materials and Methods}

The sample consisted of nine male chimpanzees at the KS (Table 1). Six of these chimpanzees lived in a group with daily membership changes (represented as (f) in Table 1), whereas the other three chimpanzees lived in a stable male group (represented as (s) in Table 1).

\subsubsection{Sample collection}

Samples were collected at the KS between May and August 2009. Hair was cut 
Running head: Hair cortisol analysis of chimpanzees

from the arms of the chimpanzees at the KS for the first time in May 2009 (Table 1, Fig. 1). We then waited for the growth of the new hair and collected hair from the same place in August 2009. Hair was cut by a caregiver (YM) with whom the subject chimpanzees had had extensive experience. We used scissors to reduce the stress of the sample collections. The chimpanzees voluntarily stretched out their arms and enabled us to cut near the bottom part of the hair, close to the skin surface. Fecal samples were collected periodically from individual indoor rooms in the mornings between May and July 2009. Behavioral data were obtained for 48 days between May 28th and July 31st 2009 by a researcher (NM) observing the group while group members changed daily. Sixty-minute observations were performed once per day from around 9:00-9:30 a.m. when the chimpanzees were introduced into the outdoor compound. The occurrence of aggressive behaviors including chase, charge, charging display, and attack were recorded using the focal-animal sampling method. The focal individuals were scored when initiating or receiving aggressive behaviors. The chimpanzees who were the recipients of aggression often showed responses such as scream and flee [39]. The number of aggressions was calculated as the number of bouts in which aggressive behaviors described above were seen. If an aggression ceased and started again after a while, we considered it to be two different bouts.

\subsubsection{Sample preparation}

\subsubsection{Hair samples}

We analyzed the hair samples collected in August. Hair samples were preserved at ambient temperature and covered in aluminum foil until assayed. The method of washing the hair and extracting cortisol from it was based on Davenport et al. [15]. Hair 
Running head: Hair cortisol analysis of chimpanzees

was placed in a 15-ml tube with isopropanol and shaken gently for $2 \mathrm{~min}$. This washing procedure was repeated three times. After washing, samples were placed in a clean hood for approximately 5 days. The hair was ground using aPrecelly 24 (Bertin Technologies, $6500 \mathrm{rpm}$ for $4 \mathrm{~min}$ ), and $40 \mathrm{mg}$ of powdered samples were weighed and placed in a 2-ml tube. One milliliter of methanol was added to each tube and incubated at ambient temperature for $24 \mathrm{hrs}$ while being shaken gently with a petite shaker (Waken B Tech). After extraction, the samples were centrifuged for $15 \mathrm{~min}$, and $0.6 \mathrm{ml}$ of supernatant was aliquoted into a clean 5-ml tube. The extracted samples were then dried at $38{ }^{\circ} \mathrm{C}$ under nitrogen gas. The samples were reconstituted with PBS buffer before assay.

\subsubsection{Fecal samples}

After collection, $1 \mathrm{~g}$ of fecal sample (wet weight) was placed in a $15-\mathrm{ml}$ tube with $4 \mathrm{ml}$ of ethanol (80 \%) and shipped from KS to the PRI. Following Khan et al. [26], the samples were not kept at ambient temperature for more than 2 weeks. As soon as the samples arrived at the PRI, the samples were stored in a biomedical freezer at $-30{ }^{\circ} \mathrm{C}$. The samples were dried at $38^{\circ} \mathrm{C}$ under nitrogen gas just before extraction. The extraction was performed based on the protocol for orangutan fecal cortisol extraction created by Yamazaki et al. [59]. Dried samples were weighed and thoroughly mixed with $5 \mathrm{ml}$ of PBS buffer for $45 \mathrm{~min}$. After being centrifuged for $15 \mathrm{~min}, 1.5 \mathrm{ml}$ of supernatant was transferred to a clean glass tube. Three milliliters of di ethylether was added and then mixed for $15 \mathrm{~min}$. Two milliliters of supernatant was evaporated using a vacuum dryer and then reconstituted with PBS buffer before assay.

\subsubsection{Cortisol Assay}


Running head: Hair cortisol analysis of chimpanzees immunosorbentassay (ELISA) based on Suzuki et al. [54]. Reagents including cortisol (hydrocortisone) used as a standard, peroxidase-labeled cortisol, and O-phenyilenediamine were purchased from Sigma (Tokyo, Japan). The antibody to cortisol was obtained from Cosmo Bio Co. (Tokyo, Japan). Microplates were obtained from Gleiner (Tokyo, Japan). The software used for the calculation was LS-Plate manager 2000 and 2004. Any duplicates with a CV greater than $20 \%$ were removed from analysis. This resulted in some variability in the number of samples used for each individual (7-10 fecal samples per each subject). Intra- and inter-assay variability for fecal cortisol assays were $15.7 \%$ and $28.3 \%$, respectively, and those for hair cortisol were $6.8 \%$ and $14 \%$, respectively.

\subsubsection{Statistical Analysis}

Parallelism was determined by analysis of covariance (ANCOVA). When the interaction between absorbance and group (standard or sample) was not significant, we considered parallelism confirmed. For fecal samples, cortisol concentration per $1 \mathrm{~g}$ dry matter was calculated by dividing cortisol concentration by dry matter weight. The numbers of initiated and received aggressions per day were calculated and used for the analysis. To check the correlations between fecal and hair cortisol concentrations and between hair cortisol concentration and aggression, we used Spearman's rank correlation test. The software used for analysis was R 2.15.1 [46] .

\subsection{Results}

\subsubsection{Parallelism test}


Running head: Hair cortisol analysis of chimpanzees

diluted fecal and hair cortisol concentrations were both very similar to those on the $F=0.030, p=0.286)$.

\subsubsection{Correlation between hair and fecal cortisol concentration}

There was no significant correlation between overall hair and mean fecal cortisol concentration (Fig. 3-a: $\mathrm{r}_{\mathrm{s}}=0.167, p=0.678, \mathrm{n}=9$ ). However, the individual with the highest hair cortisol concentration also had the highest fecal cortisol concentration.

\subsubsection{Correlation between behaviors and cortisol concentrations}

A significant correlation between number of received aggressions and hair cortisol concentration was observed (Fig. 3-b: $r_{\mathrm{s}}=0.841, p=0.036, n=6$ ). However, no significant association between number of initiated aggressions and hair cortisol concentration was found $\left(r_{\mathrm{s}}=-0.559, p=0.249, n=6\right)$. There was no significant correlation between the rate of aggressive behavior and fecal cortisol concentration (received aggressions: $r_{\mathrm{s}}=0.319, p=0.538, n=6$; initiated aggressions: $r_{\mathrm{s}}=-0.588, p=$ $0.219, n=6)$.

\section{Experiment 2}

To establish a practical methodology, we investigated the effects of body part, hair length, hair color, age, sex, and institutions on hair cortisol concentrations in Experiment 2. Additionally, we compared two different assay methodologies. 
Running head: Hair cortisol analysis of chimpanzees

\subsection{Materials and Methods}

251

252

253

254

255

256

257

258

259

260

261

262

263

264

265

266

267

268

269

270

271

272

\subsubsection{Study sites and subjects}

The sample consisted of 25 chimpanzees at the PRI and the KS (Table 1).

\subsubsection{Sample collection}

\subsubsection{Hair sample collection}

From November to December 2011, hair was cut from three parts of the bodies (arm, side, and back) of subjects at the PRI and the KS (Fig. 1). We chose these three body parts because hair loss was least evident on the back and the side, and the arm was the easiest part from which to collect hair. The hair loss level by body part are shown in supplementary materials. Samples were collected in essentially the same way as they were in Experiment 1.It was not possible to collect samples from all three parts of all individuals at KS (Table 1). To check the length of hair, YY measured three randomly chosen hairs from each sample before washing. The average length of these three hairs was used as the mean length of each hair sample.

\subsubsection{Effects of white hair}

To check the effects of white hair on cortisol concentration, we chose six mixed-color samples that had been collected from the backs or sides of PRI individuals. We divided one sample into black and white sections and then processed and analyzed them separately. Additionally, YY rated the hair color of each individual by direct observation (see supplementary materials). 
Running head: Hair cortisol analysis of chimpanzees

\subsubsection{Cortisol in distal and proximal sections of the hair shaft}

To check whether cortisol concentrations in the distal and proximal sections of the hair shaft differed, we randomly chose 10 hair samples collected from the arms or sides of KS and PRI chimpanzees. We divided each hair into two parts by approximate distance from skin surface and then processed and analyzed them separately.

\subsubsection{Sample preparation}

Hair sample storage and preparation methods were the same as those described for Experiment 1.

\subsubsection{Cortisol Assay}

We used a commercially available EIA kit (expanded range, high-sensitivity salivary cortisol EIA, Salimetrics LLC) and followed the manufacturer's instructions for the assay. We changed the assay method because the antibody used for Experiment 1 was no longer commercially available, and the Salimetric kit can reduce the time needed for assay. Intra- and inter-assay variability were $6.9 \%$ and $6.0 \%$, respectively.

\subsubsection{Statistical Analysis}

Parallelism was determined by analysis of covariance (ANCOVA). When the interaction between OD and group (standard or sample) was not significant, parallelism was confirmed. We used analysis of variance (ANOVA) to test for variations in the hair lengths of the three body parts. To check the effect of physiological factors and home institutes on cortisol concentrations, we used arepeated-measures ANCOVA and included body part, hair length, hair color, age, sex, and institute as fixed factors. To 
Running head: Hair cortisol analysis of chimpanzees

compare the hair cortisol concentration between white and black hair obtained from the same body region, we used the paired $\mathrm{t}$ test. When data were not normally distributed, we log-transformed and then Z-transformed them to achieve approximate normality. Spearman's correlation was used to check the correlations among the hair cortisol levels in the three parts of the body. The software used for analysis was R 2.15.1 [46].

\subsubsection{Parallelism}

\subsection{Results}

\subsubsection{Comparison of the assay methodologies used in Experiment 1 and Experiment 2}

To check the correlation between the two assay systems used for Experiments 1 and 2, we analyzed cortisol concentrations in the same hair samples using the two methods. We found a significant correlation between the hair cortisol concentrations calculated using the two systems $(r=0.98, p<0.001, n=8)$, although the exact mean values obtainedusing the two systems differed: $22.7 \mathrm{pg} / \mathrm{mg}$ for the Salimetric kit and $51.5 \mathrm{pg} / \mathrm{mg}$ for the assay system used in Experiment 1.

\subsubsection{Basic hair information}

Mean hair length differed among the three parts of the body (arm: $5.86 \pm 1.03$ $\mathrm{cm}$, side $5.52 \pm 0.75 \mathrm{~cm}$, back: $4.67 \pm 1.25 \mathrm{~cm}, F=11.68, p<0.001)$. Tukey’s HSD revealed that hairs taken from the arm and side were longer than were those taken from the back (arm: diff $=1.19, p<0.001$; side: diff $=0.85, p<0.01)$. Hairs taken from the 
Running head: Hair cortisol analysis of chimpanzees

321

322

323

324

back varied more in length than did hairs taken from the arm and side. Hair color information is summarized in Supp. 1-b.

\subsubsection{Effects of body part, sex, age and environmental differences on hair cortisol}

\section{concentration}

There was a significant difference in the cortisol concentrations in hair samples taken from the three parts of the body (Fig. 4: $F=7.83, P<0.01$ ). Tukey's HSD revealed that the cortisol concentrations in hairs taken from the side were significantly higher than were those taken from the arm (diff $=0.593, P<0.01$ ), and the cortisol concentrations in hairs taken from the back tended to be higher than those in hairs taken from the arm (diff $=0.414, P=0.051)$. The cortisol concentrations in hairs taken from the back and side did not differ significantly (diff $=0.179, P=0.557)$. Correlations between the cortisol concentrations in different body parts were significant, but correlations between those in hairs taken from the back and those in hairs taken from other parts were weaker than was the correlation between those in hairs taken from the arm and those taken from the side (arm-side: $r_{\mathrm{s}}=0.708, \mathrm{n}=20, p<0.001$; side-back: $r_{\mathrm{s}}$ $=0.641, n=19, p<0.01$; arm-back: $\left.r_{\mathrm{s}}=0.610, \mathrm{n}=20, p<0.01\right)$. Cortisol concentrations were higher in the KS than in the PRI chimpanzees $(F=6.14, p=0.018)$. Hair length, hair color, age, and sex did not significantly influence hair cortisol (hair length: $F=$ $0.878, p=0.355$; hair color: $F=0.003, p=0.954$; age: $F=2.688, p=0.11$; sex: $F=$ $0.478, p=0.494)$

\subsubsection{Effects of white hair on hair cortisol concentration}

Comparisons of the cortisol concentrations in white and black hairs showed 
Running head: Hair cortisol analysis of chimpanzees

that the concentrations in white hairs were consistently higher than those in black hairs (white hair: $26.5 \pm 6.91 \mathrm{pg} / \mathrm{mg}$ hair; black hair: $12.6 \pm 3.40 \mathrm{pg} / \mathrm{mg}$ hair; $t=5.39, p=$ $0.003, n=6)$.

\subsubsection{Cortisol in distal and proximal sections of hair shaft}

There were variations in the direction of the differences in the cortisol concentrations found in proximal and distal sections of hair shafts. Cortisol concentration in the distal section was higher than that in the proximal section in four of 10 samples, whereas the difference was in the reverse direction or not significant in the remaining six samples (Fig. 5).

\subsubsection{Comparison of hair cortisol concentrations between 2009 and 2011}

The cortisol concentration for Toon, the chimpanzee with the highest hair cortisol level, decreased in 2011 from its level in 2009 (51.1pg/mg in 2009; $21.5 \mathrm{pg} / \mathrm{mg}$ in 2011). In contrast, the cortisol concentration for George increased $(8.25 \mathrm{pg} / \mathrm{mg}$ in $2009 ; 28.6 \mathrm{pg} / \mathrm{mg}$ in 2011). Analysis of veterinary and daily chimpanzee care records revealed that Toon did not sustain any injury requiring medical treatment after 2010 . Additionally, the increased cortisol concentration in George matched a period during which he refused to leave his night cage. The concentration in other individuals did not vary so muchover time(average $12.7 \pm 5.29 \mathrm{pg} / \mathrm{mg}$ in 2009 ; average $16.6 \pm 3.87$ $\mathrm{pg} / \mathrm{mg}$ in 2011).

\section{Discussion}

The hair cortisol concentrations of chimpanzees can be assessed in a 
Running head: Hair cortisol analysis of chimpanzees

meaningful way. A significant correlation between hair cortisol concentration and number of received aggressions was observed here. Muller and Wrangham[40] found that urinary cortisol level and rate of aggressive behavior were positively correlated. They also found that aggressive behavior and rank were positively correlated. This is opposite to the trend found in the current study, but it is not improbable because variations in the relationship between rank and stress have been reported among primate species, and these variations may be related to factors such as availability of social supports [1]. The subject group in the present study was relatively new, and the individual with the highest hair cortisol level was one of the two individuals introduced into the group a year before the study. Therefore, hair cortisol may be a good measure of long-term stress in chimpanzees as well as in previously studied species. Nevertheless, it was difficult to precisely determine the particular stressful period that was reflected in hair cortisol levels. More detailed studies are needed to confirm the accuracy of hair cortisol as a marker of stress and to precisely determine the period of stress reflected by hair cortisol.

We did not find any significant correlation between hair and fecal cortisol concentrations. There was no significant correlation between aggressive behavior and fecal cortisol concentration. Although no previous reports on the relationship between fecal cortisol and aggressive behavior in chimpanzees have been published, such an association has been found in other animal species [e.g. 7, 14]. Additionally, changes in fecal cortisol have been reported to be associated with stressful events (anesthesia, relocation) in captive chimpanzees $[48,57]$. Given that the collection period of fecal samples matched with the period of the hair growth, sampling procedures are not likely to influence cortisol concentrations. Therefore, it may have been also due to our method 
Running head: Hair cortisol analysis of chimpanzees

of fecal cortisol analysis that there was no significant correlation between hair and fecal cortisol concentrations. The method of fecal cortisol measurement should be refined to obtain more accurate results, given that the inter-assay variability was relatively higher than that in previous studies. Nevertheless, the individual with the highest hair cortisol concentration also had the highest fecal cortisol concentration. In addition, the differences between individuals were greater than inter-assay variability. Thus, an increased sample size may have resulted in more positive results, as in a study in dogs and cats [2]. However, considering the practical limitation on the number of fecal samples available and daily changes in cortisol level, our results might mean that hair cortisol would be a better tool to assess long-term accumulation of stress.

We cut hairs from chimpanzees rather than shaving them to reduce the stress of sample collection. Chimpanzees voluntarily stretched their arms or showed their arms, sides, or backs after being asked to do so by the person who cut their hair. In contrast with shaving, which usually requires anesthesia in non-human primates, this method allowed us to collect samples more frequently without compromising subject welfare $[15,18,31]$. Although hair cortisol has been reported to reflect long-term accumulation of cortisol in many animal species, some studies reported the possibility of short-term production of hair cortisol in hair follicles, which might indicate that sample collection can influence the hair cortisol level $[25,52]$. Thus, it is important to reduce the stress of sample collection as much as possible.

We found that hair cortisol concentration varied depending on the body part from which hairs were cut. Cortisol concentrations in hairs taken from the side were higher than were those in hairs taken from the arm.However, a strong correlation was found between the cortisol concentrations of hairs taken from the side and those taken 
Running head: Hair cortisol analysis of chimpanzees

417 from the arm; thus, the trends characterizing changes in the cortisol levels measured 418 from these two body parts may be similar. Macbeth et al. (2010) also found 419 intra-individual variations in hair cortisol concentration in grizzly bears. They 420 considered that this variations was due to differences in timing and patterns of hair 421 growth in individuals. The cortisol concentrations in hairs taken from the side and back 422 did not differ significantly, but the strength of this correlation was weaker than that 423 between hairs taken from the side and those taken from the arm. Hair length varied among individuals, and the variations between individuals were most evident on the back. Thus, similarly to grizzly bears, chimpanzees might also show intra-individual variations in hair cortisol concentration due to such differences in timing and patterns of hair growth.

In addition, variations in hair color across body parts can also influence the regional differences. This is because white hair was more evident on the back and higher cortisol concentrations were found in white than in black hair, even when collected from the same body part. Although hair color was not a significant factor in the final model, it may be that the rating system used to assess black hair levels was not sensitive enough to detect a relationship between hair cortisol and hair color. Supporting the importance of this factor, Bennett et al. [9] also found that cortisol concentrations differed in relation to coat color in dogs. They discussed the possible roles of stress-associated inhibition of hair growth as well as of melanocyte development and differentiation. However, it is also possible that white and black hair differ with respect to the efficiency with which cortisol can be extracted given that it is well known that melanin can inhibit the efficiency of DNA extraction [60]. However, because the exact nature of this variation in chimpanzees was not clear, it is better to avoid white hair as 
Running head: Hair cortisol analysis of chimpanzees

much as possible when using hair samples as a method of cortisol measurement.

We chose these three body parts for hair cortisol analysis based on the practical convenience of collection as well as on the stable presence of hair on those sites across many individuals. Previous studies in humans and other animals have used hair from the posterior vertex region, where the lowest coefficient of variation has been found in humans $[15,22]$. However, in chimpanzees, hair loss was most evident in this region, and it was not a feasible sample-collection site. Based on the results of this study, the sides or arms may provide the best hair samples for chimpanzees.

Proximal-distal comparisons revealed that individuals varied in terms of the direction of the differences between the cortisol concentrations in hairs taken from the proximal section and those taken from the distal section of the same hair samples. It is hypothesized that because cortisol accumulates during hair growth, different sections of hair differ in this regard. The results of this study support this hypothesis because the direction of difference would be more consistent if cortisol concentrations varied according to more basic factors such as absolute amount of time elapsed. Other animal studies found no significant differences in hair cortisol level between proximal and distal sections of hair $[9,15,32]$. Although some studies in humans also did not find a time-dependent change in hair cortisol level as in animal studies, other studies found a continuous decrease in hair cortisol in older hair segments [21, 27, 33]. However, in general, a significant decrease in hair cortisol concentration is most obvious beyond $6 \mathrm{~cm}$ distal from scalp [49]. Chimpanzee hairs examined were not as long as those in humans, which might be one reason that there were no systematic change in hair cortisol according to the distance from the scalp.Sex, age, and hair length did not significantly affect cortisol concentration. However, different results may have been 
Running head: Hair cortisol analysis of chimpanzees

obtained if we had included samples from younger individuals. A previous study found differences in hair cortisol concentrations among different age groups of vervet monkeys [31]. Future research should analyze hair samples from more individuals across a wider range of age groups.

We found that cortisol levels tended to be higher in the KS group, which is not surprising considering the fact that these individuals lived in an all-male group, and the social composition of the group changed on a daily basis. However, this does not necessarily mean that the welfare of the chimpanzees at the KS was worse. Indeed, all-male groups can be an alternative way of supplying social stimulation when it is difficult to keep several males in a mixed-sex group. In addition, wild chimpanzee societies are characterized by patterns of fission and fusion according to which group companions change. For this reason, the manipulation of group membership may be a good way to provide chimpanzees with a more stimulating life in a captive environment. Nevertheless, effects of these husbandry procedures on stress in chimpanzees have rarely been documented and it was not clear whether the challenges posed to the chimpanzees by these social management schemes were at an appropriate level [11, 37, 42]. Toon's cortisol concentration in 2009 was much higher than that of other individuals, but it decreased to a more typical level in 2011. This large change over time suggests that long-term analysis of hair cortisol and comparisons among multiple individuals from different social environments are necessary to understand the effects of all-male group and social variability. From this perspective, hair cortisol has potentially greater value than other methods that have reported stability over several years $[8,56]$. Assessment of long-term stress is important from the view of animal welfare, because long-term stress can have negative effects on welfare including behavioral 
Running head: Hair cortisol analysis of chimpanzees

489

490

491

492

493

494

495

496

497

498

499

500

501

502

503

504

505

506

507

508

509

510

511

512

changes, suppression of reproduction and increase of the likelihood of becoming sick

$[11,13,53]$. The link between long-term stress and those welfare parameters has rarely been investigated in chimpanzees. However, there are some problems in captive chimpanzee care which can be related to long-term stress. For example, one of the leading causes of death in captive great apes is heart disease, of which the actual cause is not well understood $[51,55]$. In humans, it is said that both short-term and long-term stress can increase the risk of developing heart disease [16]. Furthermore, maternal rejection and inappropriate care of infants are major problems in captive chimpanzees, and studies have shown that stress might impair maternal behaviors $[6,50]$. Therefore, investigating long-term stress might shed light on the unknown causes of these important issues.

In conclusion, hair cortisol measurement is a promising tool for estimating long-term stress in chimpanzees. Hair collection should be made from the arm or side while avoiding white hair as much as possible. In combination with behavioral measures, this approach can contribute to understanding the long-term effects of husbandry procedures on the welfare of chimpanzees.

\section{Acknowledgement}

This study was financially supported by grants from MEXT Japan (\#20002001 and \#24000001) to Tetsuro Matsuzawa. It was also supported by grants from the Japan Society for Promotion of Science (\#10J05294 to YY, \#19700245 and \#23700313 to $\mathrm{MH}$ ) and by the Grants to Excellent Graduate Schools program of MEXT.

We are very grateful to the following people who supported our study: Tetsuro Matsuzawa, Masaki Tomonaga, Ikuma Adachi, Satoshi Hirata, Shohei Watanabe, 
Running head: Hair cortisol analysis of chimpanzees

513 KiyonoriKumazaki, Norihiko Maeda and the members of the Language and Intelligence

514 Section and the Center for Human Evolution Model Research of the PRI. We thank

515 Tomoko Takashima and Etsuko Ichino for their help in assessing basic hair information.

516 We thank Chris Martin, Masayuki Tanaka, Michael Seres and Elizabeth Nakajima for

517 checking an earlier draft of this manuscript. 
Running head: Hair cortisol analysis of chimpanzees

\section{References}

[1] D.H. Abbott, E.B. Keverne, F.B. Bercovitch, C.A. Shively, S.P. Medoza, W. Saltzman, et al., Are subordinates always stressed? A comparative analysis of rank differences in cortisol levels among primates. Hormones and Behavior. 43 (2003) 67-82.

[2] P.A. Accorsi, E. Carloni, P. Valsecchi, R. Viggiani, M. Garnberoni, C. Tarnanini, et al., Cortisol determination in hair and faeces from domestic cats and dogs. General and Comparative Endocrinology. 155 (2008) 398-402.

[3] S.F. Anestis, R.G. Bribiescas, Rapid changes in chimpanzee (Pan troglodytes) urinary cortisol excretion. Hormones and Behavior. 45 (2004) 209-213.

[4] S.F. Anestis, R.G. Bribiescas, D.L. Hasselschwert, Age, rank, and personality effects on the cortisol sedation stress response in young chimpanzees. Physiology \& Behavior. 89 (2006) 287-294.

[5] N.I. Bahr, R. Palme, U. Mohle, J.K. Hodges, M. Heistermann, Comparative aspects of the metabolism and excretion of cortisol in three individual nonhuman primates. General and Comparative Endocrinology. 117 (2000) 427-438.

[6] N.I. Bahr, C.R. Pryce, M. Döbeli, R.D. Martin, Evidence from urinary cortisol that maternal behavior is related to stress in gorillas1. Physiology \& Behavior. 64 (1998) 429-437.

[7] G.M. Barrett, K. Shimizu, M. Bardi, S. Asaba, A. Mori, Endocrine correlates of rank, reproduction, and female-directed aggression in male Japanese macaques (Macaca fuscata). Hormones and Behavior. 42 (2002) 85-96.

[8] T.Ø. Bechshøft, F.F. Rigét, C. Sonne, R.J. Letcher, D.C.G. Muir, M.A. Novak, et al., Measuring environmental stress in East Greenland polar bears, 1892-1927 and 1988-2009: What does hair cortisol tell us? Environment International. 45 (2012) 15-21.

[9] A. Bennett, V. Hayssen, Measuring cortisol in hair and saliva from dogs: coat color and pigment differences. Domestic Animal Endocrinology. 39 (2010) 171-180.

[10] M.A. Bloomsmith, K.C. Baker, Social management of captive chimpanzees. In: L. Brent, (Ed.), The care and management of captive chimpanzees, American Society of Primatologists, San Antonio, 2001, pp. 205-242.

[11] D.M. Broom, K.G. Johnson, Stress and animal welfare, Chapman \& Hall: London, 1993.

[12] F. Carlsen, European studbook for the chimpanzees. (2009). 
Running head: Hair cortisol analysis of chimpanzees

553

554

555

556

557

558

559

560

561

562

563

564

565

566

567

568

569

570

571

572

573

574

575

576

577

578

579

580

581

582

583

584

585

586

587

588

[13] K. Carlstead, Shepherdson, D., Effects of environmental enrichment on reproduction. Zoo Biology. 13 (1994) 447-458.

[14] S.A. Cavigelli, Behavioural patterns associated with faecal cortisol levels in free-ranging female ring-tailed lemurs,Lemur catta. Animal Behaviour. 57 (1999) 935-944.

[15] M.D. Davenport, S. Tiefenbacher, C.K. Lutz, M.A. Novak, J.S. Meyer, Analysis of endogenous cortisol concentrations in the hair of rhesus macaques. General and Comparative Endocrinology. 147 (2006) 255-261.

[16] J.E. Dimsdale, Psychological stress and cardiovascular disease. Journal of the American College of Cardiology. 51 (2008) 1237-1246.

[17] M. Emery Thompson, M.N. Muller, S.M. Kahlenberg, R.W. Wrangham, Dynamics of social and energetic stress in wild female chimpanzees. Hormones and Behavior. 58 (2010) 440-449.

[18] L.A. Fairbanks, M.J. Jorgensen, J.N. Bailey, S.E. Breidenthal, R. Grzywa, M.L. Laudenslager, Heritability and genetic correlation of hair cortisol in vervet monkeys in low and higher stress environments. Psychoneuroendocrinology. 36 (2011) 1201-1208.

[19] D. Fraser, Understanding animal welfare: The science in its cultural context, Wiley Blackwell: Chichester, 2008.

[20] J. Fritz, S. Howell, Captive chimpanzee social group formation. In: L. Brent, (Ed.), The care and management of captive chimpanzees, American Society of Primatologists, San Antonio, 2001, pp. 173-204.

[21] W. Gao, Q. Xie, J. Jin, T. Qiao, H. Wang, L. Chen, et al., HPLC-FLU detection of cortisol distribution in human hair. Clinical Biochemistry. 43 (2010) 677-682.

[22] R. Gow, S. Thomson, M. Rieder, S. Van Uum, G. Koren, An assessment of cortisol analysis in hair and its clinical applications. Forensic Science International. 196 (2010) 32-37.

[23] Great Ape Information Network. http://www.shigen.nig.ac.jp/gain/top.jsp.

[24] M.R. Heintz, R.M. Santymire, L.A. Parr, E.V. Lonsdorf, Validation of a cortisol enzyme immunoassay and characterization of salivary cortisol circadian rhythm in chimpanzees (Pan troglodytes). American Journal of Primatology (2011) 903-908.

[25] N. Ito, T. Ito, A. Kromminga, A. Bettermann, M. Takigawa, F. Kees, et al., Human hair follicles display a functional equivalent of the hypothalamic-pituitary-adrenal (HPA) axis and synthesize cortisol. FASEB J. (2005) 04-1968fje. 
Running head: Hair cortisol analysis of chimpanzees

589

590

591

592

593

594

595

596

597

598

599

600

601

602

603

604

605

606

607

608

609

610

611

612

613

614

615

616

617

618

619

620

621

622

[26] M.Z. Khan, J. Altmann, S.S. Isani, J. Yu, A matter of time: evaluating the storage of fecal samples for steroid analysis. General and Comparative Endocrinology. 128 (2002) 57-64.

[27] C. Kirschbaum, A. Tietze, N. Skoluda, L. Dettenborn, Hair as a retrospective calendar of cortisol production-Increased cortisol incorporation into hair in the third trimester of pregnancy. Psychoneuroendocrinology. 34 (2009) 32-37.

[28] L. Koren, E. Geffen, Androgens and social status in female rock hyraxes. Animal Behaviour. 77 (2009) 233-238.

[29] Kumamoto Sanctuary. http://www.wrc.kyoto-u.ac.jp/kumasan/indexE.html.

[30] N. Kutsukake, K. Ikeda, S. Honma, M. Teramoto, Y. Mori, I. Hayasaka, et al., Validation of salivary cortisol and testosterone assays in chimpanzees by liquid chromatography-tandem mass spectrometry. American Journal of Primatology. 71 (2009) 696-706.

[31] M.L. Laudenslager, M.J. Jorgensen, L.A. Fairbanks, Developmental patterns of hair cortisol in male and female nonhuman primates: Lower hair cortisol levels in vervet males emerge at puberty. Psychoneuroendocrinology (2012).

[32] B.J. Macbeth, M.R.L. Cattet, G.B. Stenhouse, M.L. Gibeau, D.M. Janz, Hair cortisol concentration as a noninvasive measure of long-term stress in free-ranging grizzly bears (Ursus arctos): considerations with implications for other wildlife. Canadian Journal of Zoology-Revue Canadienne De Zoologie. 88 (2010) 935-949.

[33] L. Manenschijn, J.W. Koper, S.W.J. Lamberts, E.F.C. van Rossum, Evaluation of a method to measure long term cortisol levels. Steroids. 76 (2011) 1032-1036.

[34] T. Matsuzawa, The Ai project: historical and ecological contexts. Animal Cognition. 6 (2003) 199-211.

[35] T. Matsuzawa, Sociocognitive development in chimpanzees: a synthesis of laboratory work and fieldwork. In: T. Matsuzawa, M. Tomonaga, M. Tanaka, (Eds.), Cognitive development in chimpanzees., Springer, Tokyo, 2006, pp. 3-33.

[36] T. Matsuzawa, M. Tomonaga, M. Tanaka, Cognitive development in chimpanzees, Springer: Tokyo, 2006.

[37] C.L. Meehan, J.A. Mench, The challenge of challenge: Can problem solving opportunities enhance animal welfare? Applied Animal Behaviour Science. 102 (2007) 246-261. 
Running head: Hair cortisol analysis of chimpanzees

623

624

625

626

627

628

629

630

631

632

633

634

635

636

637

638

639

640

641

642

643

644

645

646

647

648

649

650

651

652

653

654

655

656

[38] N. Morimura, G. Idani, T. Matsuzawa, The first chimpanzee sanctuary in Japan: an attempt to care for the "surplus" of biomedical research. American Journal of Primatology. 73 (2010) 226-232.

[39] N. Morimura, Y. Mori, Effects of early rearing conditions on problem-solving skill in captive male chimpanzees (Pan troglodytes). American Journal of Primatology. 72 (2010) 626-633.

[40] M.N. Muller, R.W. Wrangham, Dominance, cortisol and stress in wild chimpanzees (Pan troglodytes schweinfurthii). Behavioral Ecology and Sociobiology. 55 (2004) 332-340.

[41] T. Ochiai, T. Matsuzawa, Planting trees in an outdoor compound of chimpanzees for an enriched environment., Third International Conference on Environmental Enrichment., Orlando, 1997, pp. 355-364.

[42] T. Ogura, S. Yamazaki, Y. Yamanashi, S. Mitsuya, Future prospects of animal welfare research. Japanese Journal of Animal Psychology. 61 (2011) 115-124.

[43] Primate Research Institute. http://langint.pri.kyoto-u.ac.jp/ai/.

[44] Project ChimpCARE. http://www.chimpcare.org/.

[45] J.D.E. Pruetz, W.C. McGrew, What does a chimpanzee need? Using natural behavior to guide the care and management of captive populations. In: L. Brent, (Ed.), Care and Management of Captive Chimpanzees., American Society of Primatologists, San Antonio, 2001, pp. 16-37.

[46] R Development Core Team, R: A language and environment for statistical computing. R Foundation for Statistical Computing, Vienna, 2011.

[47] D.M. Reeder, K.M. Kramer, Stress in free-ranging mammals: integrating physiology, ecology, and natural history. Journal of Mammalogy. 86 (2005) 225-235.

[48] M. Reimers, F. Schwarzenberger, S. Preuschoft, Rehabilitation of research chimpanzees: Stress and coping after long-term isolation. Hormones and Behavior. 51 (2007) 428-435.

[49] E. Russell, G. Koren, M. Rieder, S. Van Uum, Hair cortisol as a biological marker of chronic stress: Current status, future directions and unanswered questions. Psychoneuroendocrinology. 37 (2012) 589-601.

[50] W. Saltzman, D.H. Abbott, Effects of elevated circulating cortisol concentrations on maternal behavior in common marmoset monkeys (Callithrix jacchus). Psychoneuroendocrinology. 34 (2009) 1222-1234. 
Running head: Hair cortisol analysis of chimpanzees

657

658

659

660

661

662

663

664

665

666

667

668

669

670

671

672

673

674

675

676

677

678

679

680

681

682

683

684

685

686

687

[51] B.M. Seiler, E.J. Dick Jr, R. Guardado-Mendoza, J.L. VandeBerg, J.T. Williams, J.N. Mubiru, et al., Spontaneous heart disease in the adult chimpanzee (Pan troglodytes). Journal of Medical Primatology. 38 (2009) 51-58.

[52] C.F. Sharpley, K.G. Kauter, J.R. McFarlane, An initial exploration of in vivo hair cortisol responses to a brief pain stressor: latency, localization and independence effects. Physiological Research. 58 (2009) 757-761.

[53] J.E. Squires, Applied animal endocrinology: 2nd edtion, CAB International: Oxfordshire, 2010.

[54] J. Suzuki, S. Ohkura, K. Terao, Baseline and stress levels of cortisol in conscious and unrestrained Japanese macaques (Macaca fuscata). Journal of Medical Primatology. 31 (2002) 340-344.

[55] N. Varki, D. Anderson, J.G. Herndon, T. Pham, C.J. Gregg, M. Cheriyan, et al., Heart disease is common in humans and chimpanzees, but is caused by different pathological processes. Evolutionary Applications. 2 (2009) 101-112.

[56] E. Webb, S. Thomson, A. Nelson, C. White, G. Koren, M. Rieder, et al., Assessing individual systemic stress through cortisol analysis of archaeological hair. Journal of Archaeological Science. 37 (2010) 807-812.

[57] P.L. Whitten, R. Stavisky, F. Aureli, E. Russell, Response of fecal cortisol to stress in captive chimpanzees (Pan troglodytes). American Journal of Primatology. 44 (1998) 57-69.

[58] J. Yamada, B. Stevens, N. de Silva, S. Gibbins, J. Beyene, A. Taddio, et al., Hair cortisol as a potential biologic marker of chronic stress in hospitalized neonates. Neonatology. 92 (2007) 42-49.

[59] S. Yamazaki, J. Suzuki, H. Kurotori, S. Takeda, Y. Ueno, Behavioural and physiological impacts on Borneo Orangutans in response to introduction to a new exhibit as structural enrichment. Proceedings of the Nineth International Conference on Environmental Enrichment (2009) 132.

[60] T. Yoshii, K. Tamura, I. Ishiyama, Presence in a PCR-inhibitor in hairs. Japanese journal of legal medicine. 46 (1992) 313-316. 
Running head: Hair cortisol analysis of chimpanzees

688

Fig 1. Illustration of sample collection and the parts of the body from which hair

689 samples were collected
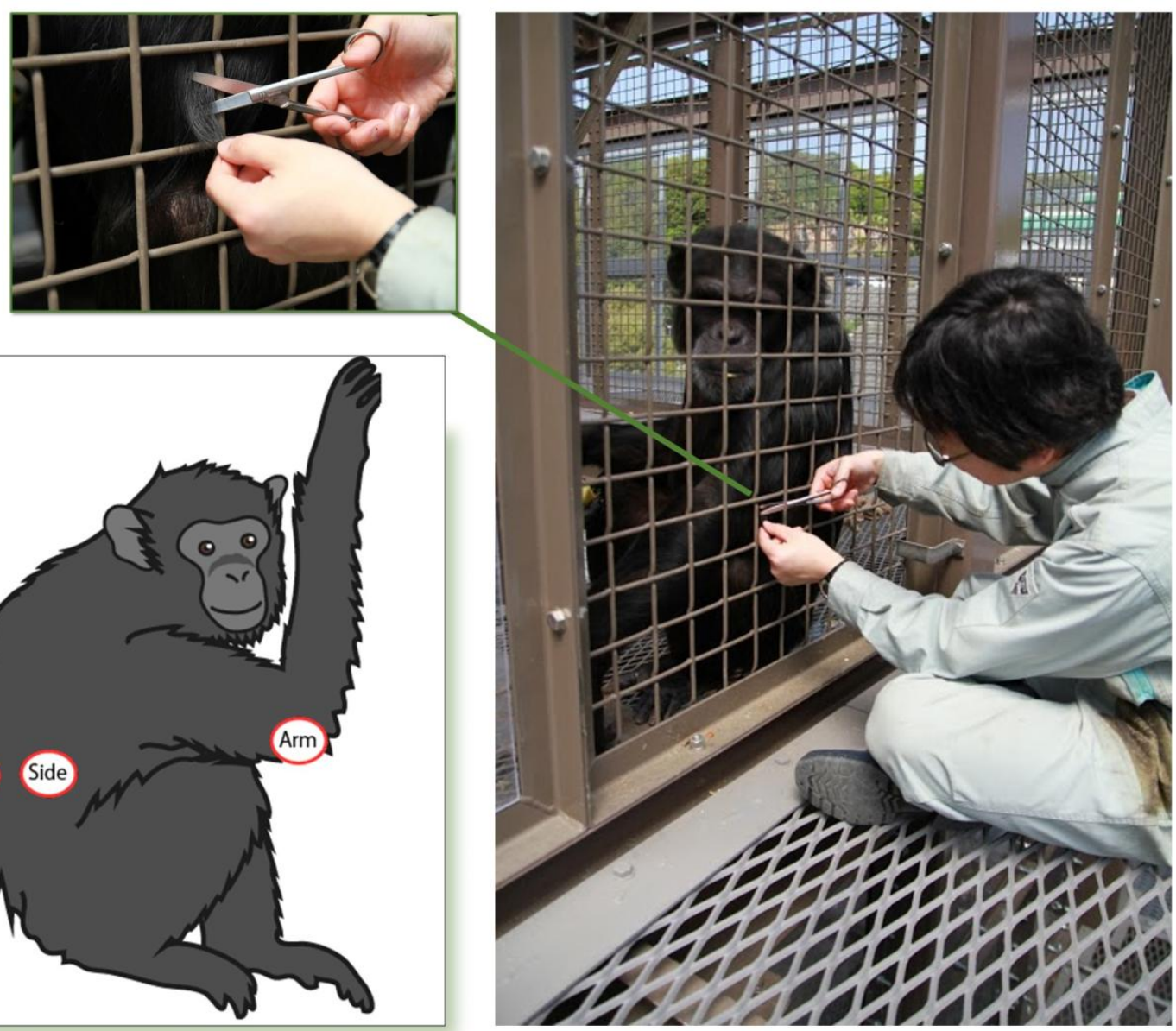

690

691 
Running head: Hair cortisol analysis of chimpanzees

692

Fig2. Parallelism for Experiment 1 and 2

693 Parallelism between cortisol standards and serially diluted samples.

694 Fig 2-a. The results of parallelism test of fecal cortisol and cortisol standards for 695 Experiment 1.

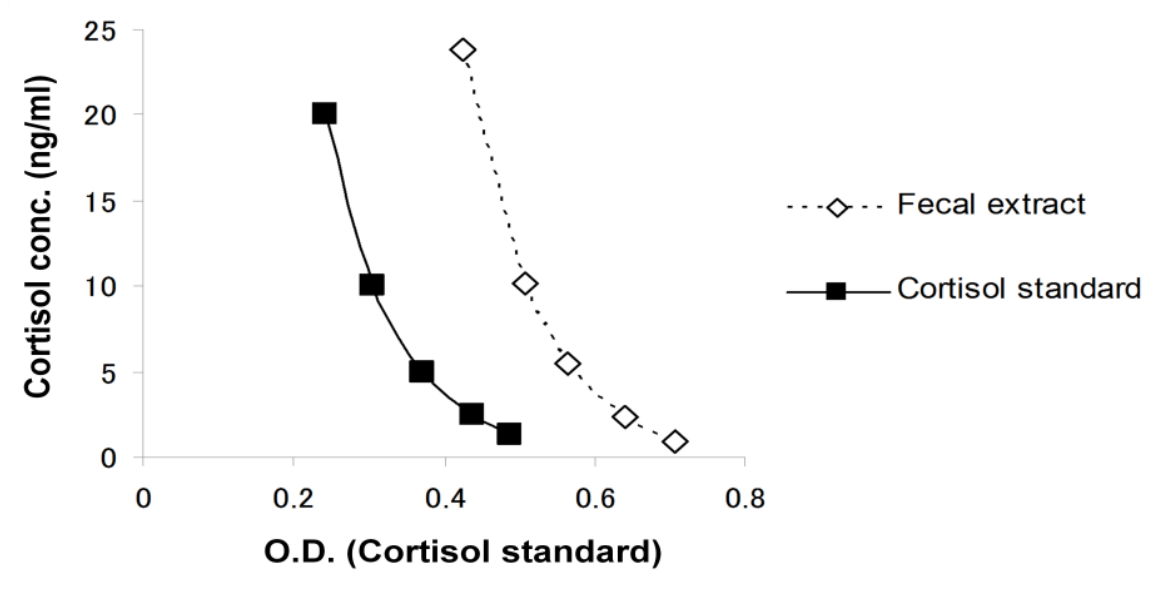

O.D. (Fecal extract)

696

697 Fig 2-b. The results of parallelism test of hair cortisol and cortisol standards for 698 Experiment 1.

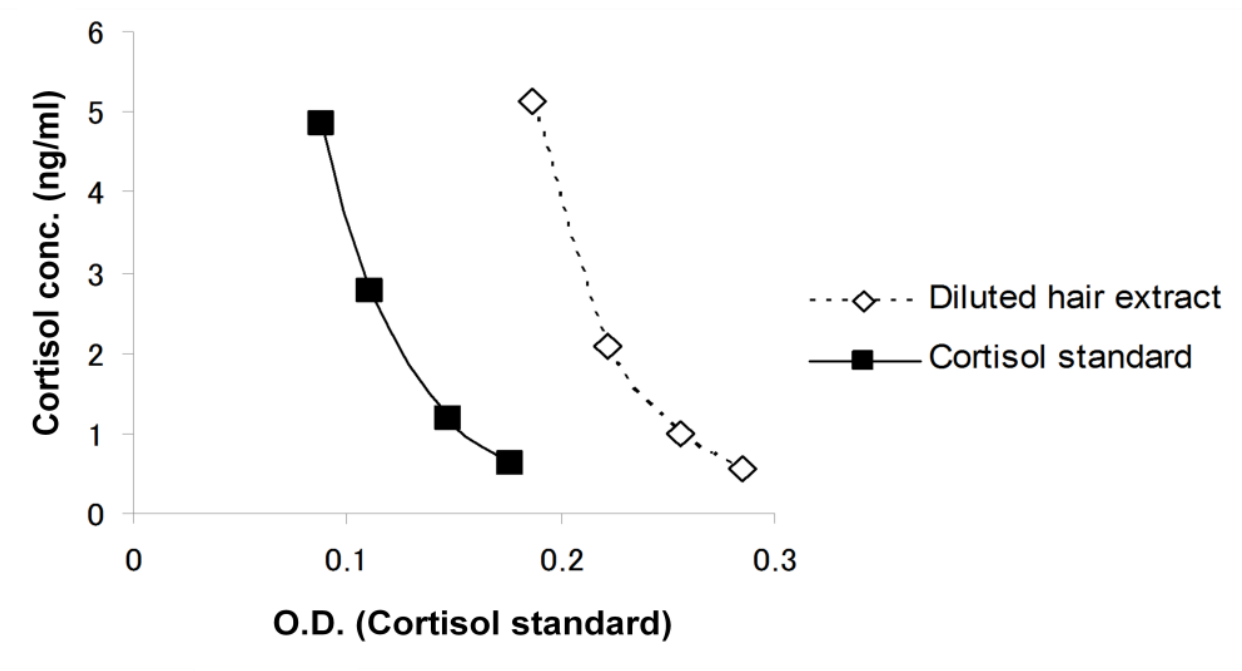

699 
Running head: Hair cortisol analysis of chimpanzees

701 Fig 3. Correlation between hair cortisol concentration and other measures

702 Fig 3-a. Correlation between fecal and hair cortisol concentration

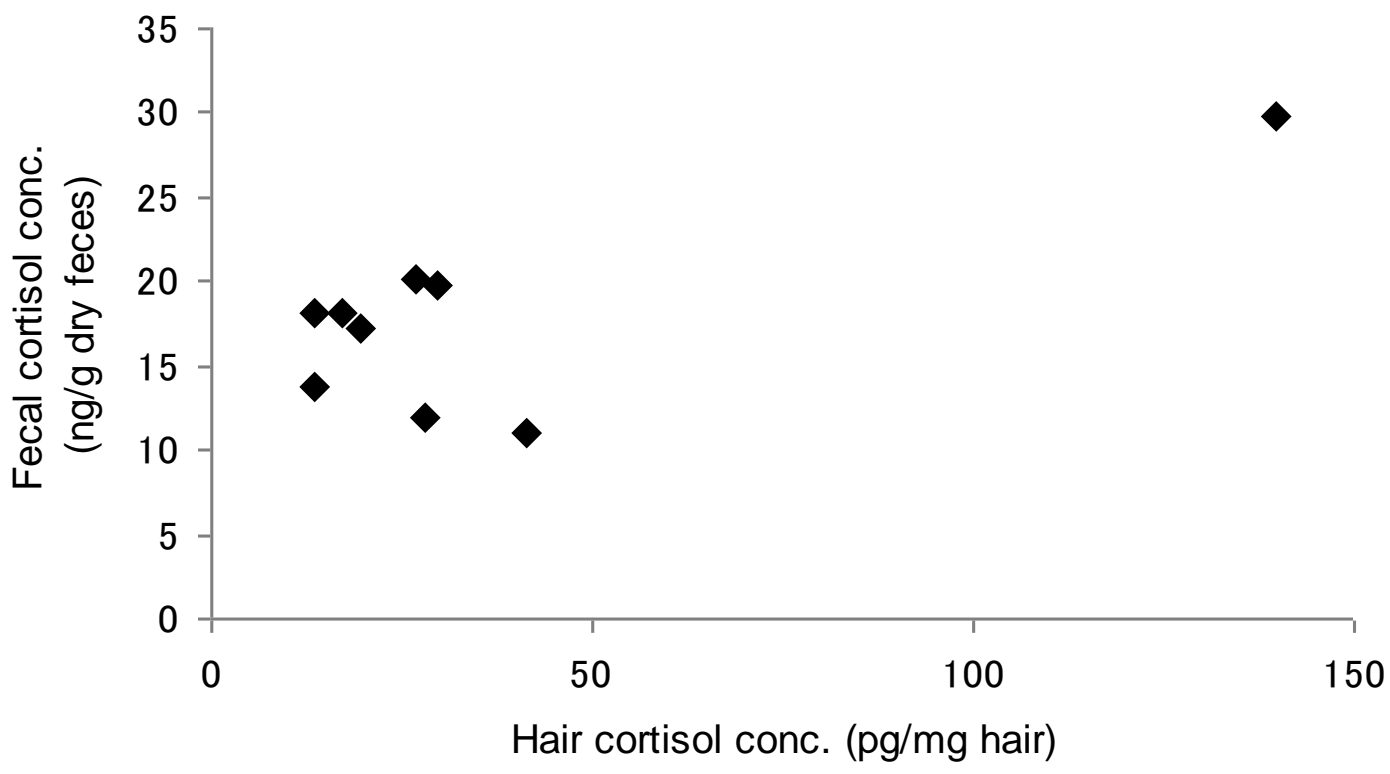

703

704 Fig 3-b. Correlation between hair cortisol concentration and the rate of receiving 705 aggression

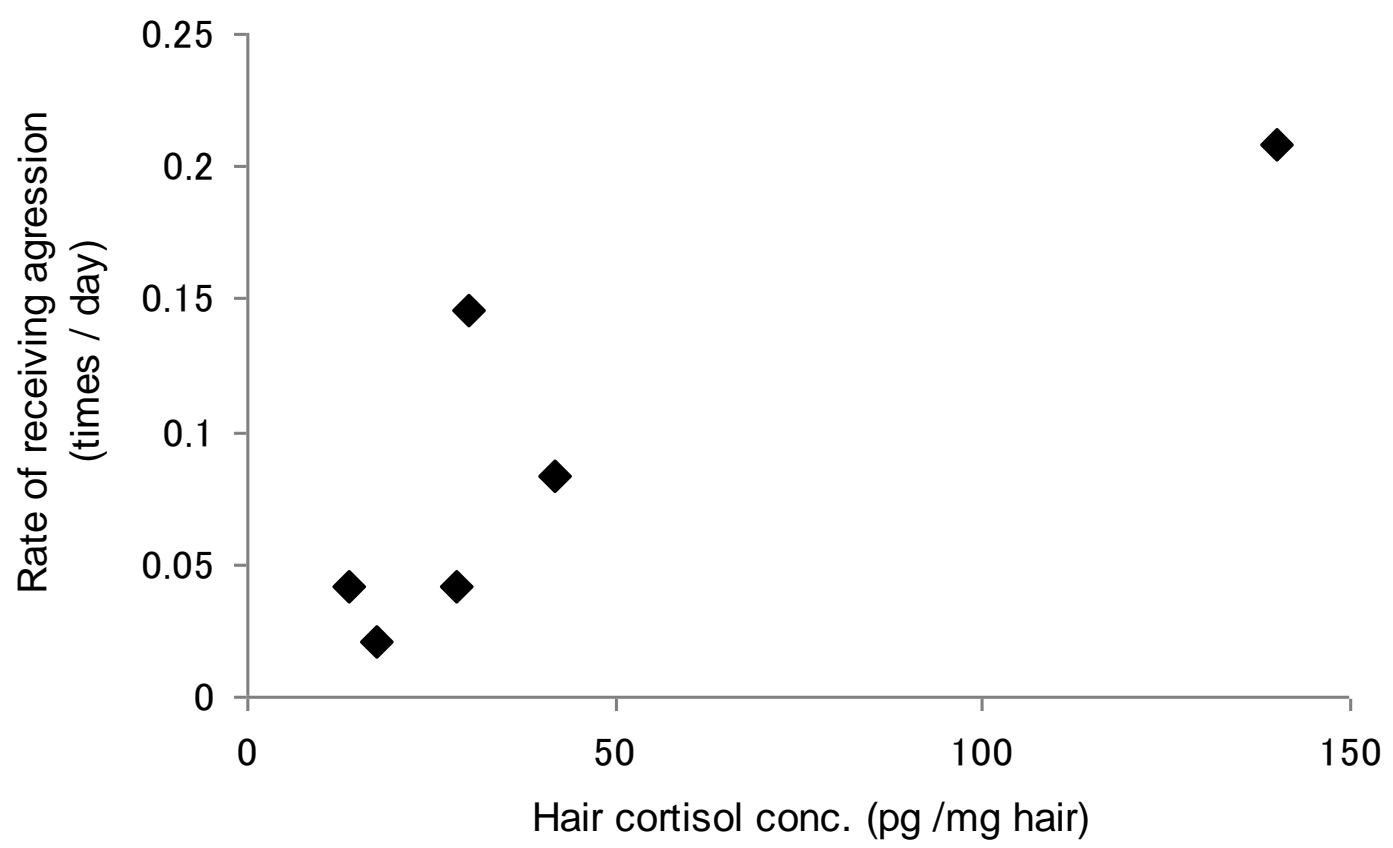


Running head: Hair cortisol analysis of chimpanzees

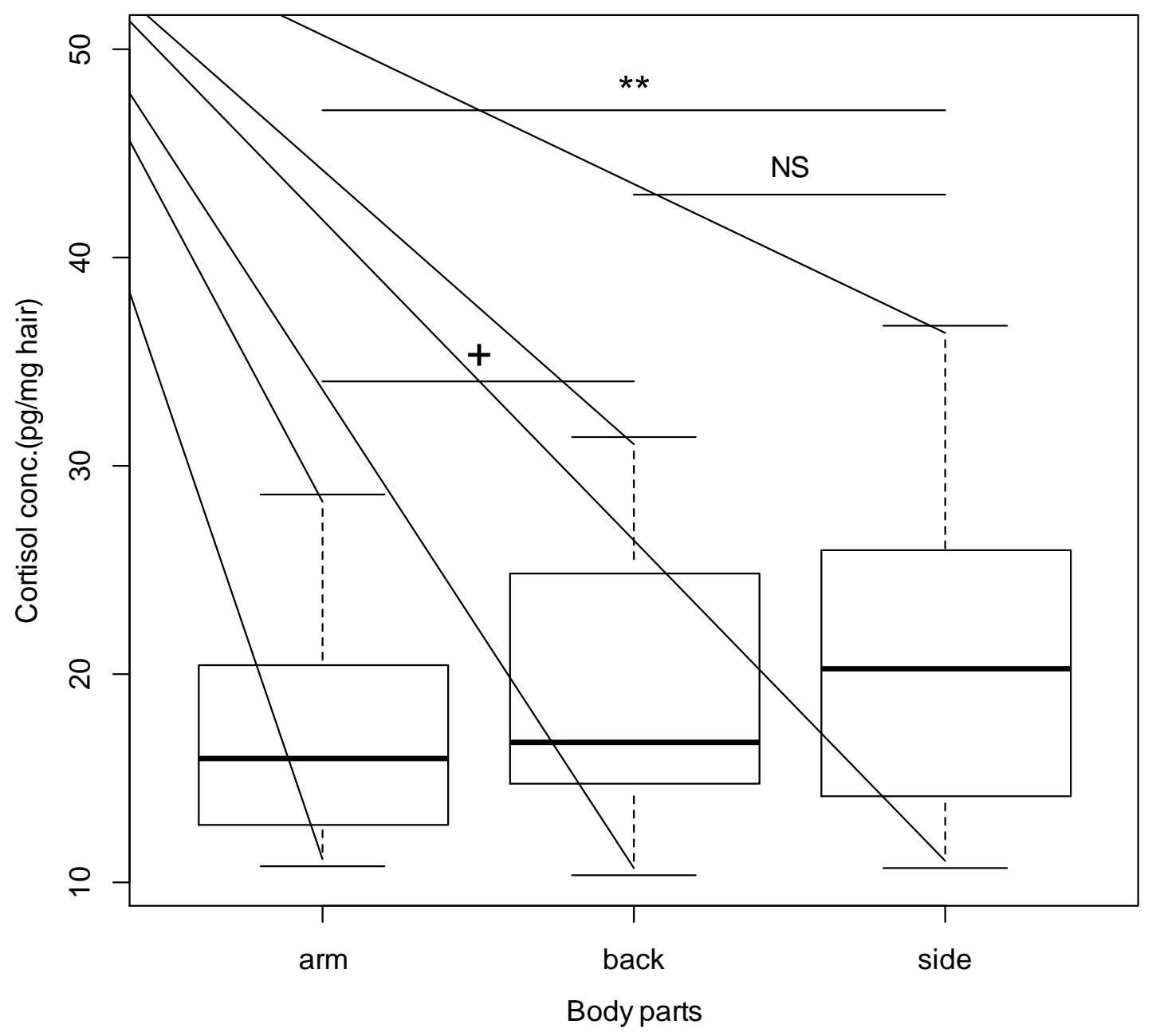

709 
Running head: Hair cortisol analysis of chimpanzees

\section{Fig 5. Proximal-distal comparison of cortisol concentration}

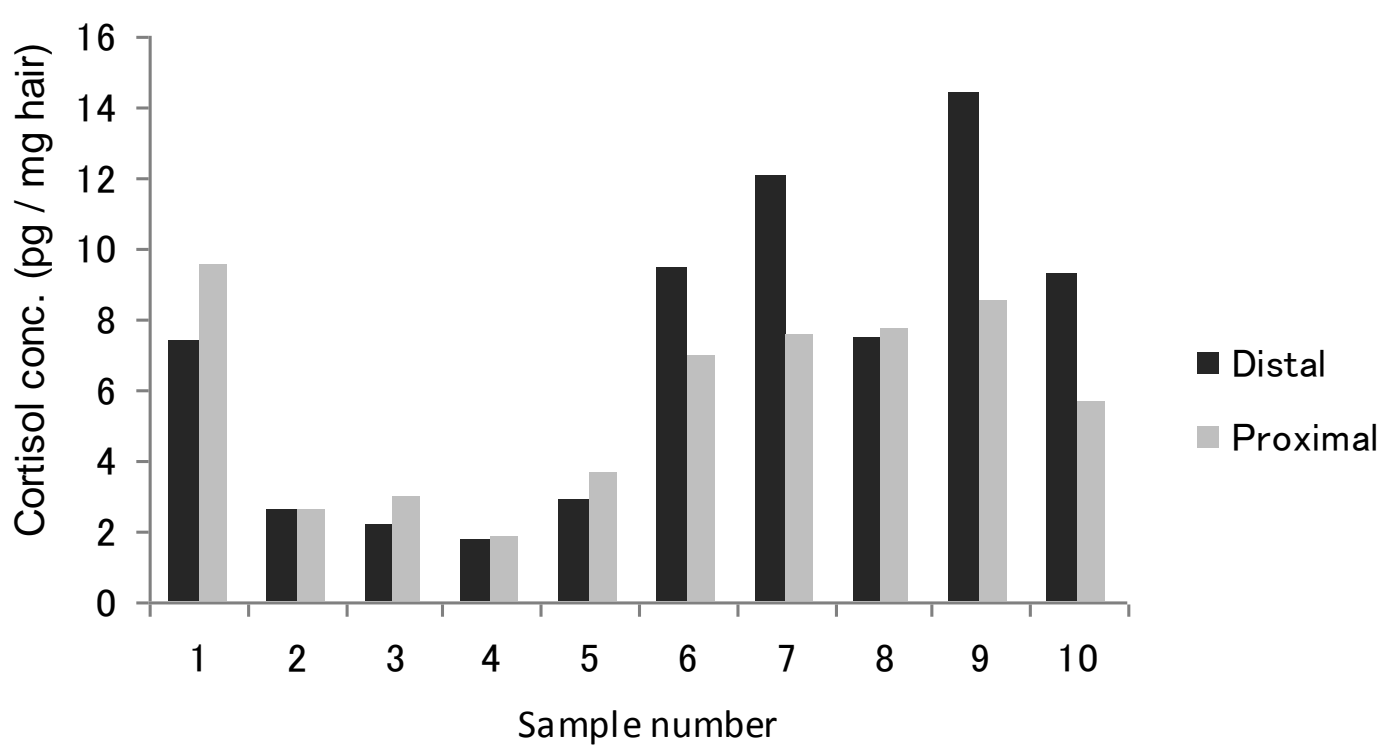

